\title{
Proteinuria in Cynomolgus macaques (Macaca fascicularis) with Spontaneously Developed Metabolic Disorder and Diabetes: Transcriptome Analysis of Biopsy Kidney

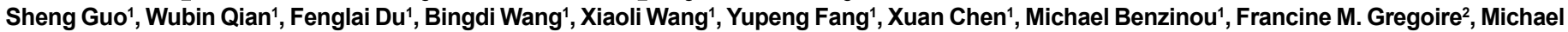 Staup ${ }^{2}$, Keefe $\mathrm{Chng}^{2}$, Yaxiong Chen ${ }^{1}$, Yong-Fu Xiao', Yi-Xin (Jim) Wang ${ }^{1,2 *}$
}

${ }^{1}$ Cardiovascular and Metabolic Diseases Research, Crown Bioscience Inc. Taicang, Jiangsu Province, China

${ }^{2}$ The International Institute of Biomedical Research, a Crown Bioscience Company at David H. Murdock Research Institute, Kannapolis, NC, USA

\begin{abstract}
Although Type 2 Diabetes Mellitus (T2DM) is well characterized Non-Human Primate (NHP), the phenotypes of diabetic nephropathy, and its molecular mechanisms are not well studied in NHPs. Diabetic nephropathy in cynomolgus macaques with spontaneous dysmetabolism (Pre-DM, $n=19)$ and diabetes $(D M, n=20)$ were compared with normal controls $(\mathrm{N}, \mathrm{n}=11)$. There were $9 \mathrm{NHPs}$ with albuminuria $(>42 \mathrm{mg} /$ day) in the DM $(45 \%)$, only 1 in Pre-DM and none in $\mathrm{N}$ group. The renal function measured by estimated glomerular filtration rate (eGFR) was not significantly different among the 3 groups, indicating that these NHPs were in an early stage of renal disease. From these NHPs, $3 \mathrm{~N}, 3$ Pre-DM without albuminuria and 6 DM with albuminuria were selected for transcriptome analysis of kidney biopsies. There were 95 differentially expressed genes (DEGs) detected amongst the 3 groups, of which, 75DEGs between the $\mathrm{N}$ and DM related to diabetic nephropathy; 66 DEGs between the $\mathrm{N}$ and Pre-DM related to dysmetabolism without nephropathy; 68 DEGs between $\mathrm{N}$ and both Pre-DM \& DM related to dsymetabolism; but only 1 nephropathy specific gene (LCT lactase) between the DM with albuminuria (DM) and Pre-DM without albuminuria; only 4 DEGs between the DM with albuminuria and both N \& Pre-DM without albuminuria specific to nephropathy. Signaling pathway analysis of the relevant DEGs and encoded proteins highlighted the role of a kidney failure, renal and urological diseases, and inflammatory diseases related network, in which the most pivotal gene in this network is Tumor Necrosis Factor (TNF), indicating that nephropathy is a disease closely related to inflammation and cell death. Thus, the present study was the first detailed characterization of the diabetic nephropathy phenotypes and the kidney histopathological changes in NHPs and provided molecular insights into novel mechanisms of disease progression, potential new drug targets as well as specific diagnostic biomarkers.
\end{abstract}

Keywords: Micro-albuminuria; Proteinuria; Glycosuria; Renal function; Kidney biopsy; Trascriptome analysis; Affymetrix genechip microarray

Abbreviations: DM: Diabetes Mellitus; Pre-DM: Prediabetic or Dysmetabolic; ivGTT: Intravenous Glucose Tolerance Test; Kglucose: Glucose Disappearance Rate; Dvolume: Total Drinking Volume in 24 hours (mL); Uvolume: Total Urine Volume in 24 hours $(\mathrm{mL})$; Uprotein: Total Urinary Protein Excretion in 24 hours (mg); Ualbumin: Total Urinary Albumin Excretion in 24 hours (mg); Ucreatinine: Total Urinary Creatinine Excretion in 24 hours (mg); ACR: Urinary Albumin and Creatinine Ratio; GFR: Glumerular Filtration Rate; BUN: Blood Urea Nitrogen Concentration; DEG: Deferentially Expressed Gene

\section{Introduction}

Long standing diabetes mellitus with characterization of nephrotic syndrome is the major cause of End-Stage Renal Disease (ESRD) that leads to dialysis in $20-40 \%$ patients with kidney disease. During the present decade, $30 \%$ of the predicted $\$ 1.1$ trillion medical costs of dialysis world-wide will result from diabetic nephropathy. A study in Diabetes Care revealed that Type 2 Diabetes Mellitus (T2DM) patients with micro-albuminuria or diabetic retinopathy were more likely to progress to macro-albuminuria and a faster decline in renal function in the late stage compared with those who did not have these conditions [1]. Treatment of T2DM patients can delay the development of micro-albuminuria, e.g., the Angiotensin II Converting Enzyme (ACE) inhibitor, trandolapril, or the angiotensin-receptor blocker, olmesartan, both have shown to delay the T2DM-related microalbuminuria [2-7]. Currently this disease is not recognized early enough because of inadequate diagnostic methods, which increases the chances that early nephropathy with micro-albuminuria will progress toward ESRD. Thus, in addition to micro-albuminuria that has been commonly used as an early biomarker of diabetic nephropathy, there is still an unmet medical need to identify novel and highly sensitive biomarkers for detecting diabetic nephropathy at an early stage, and possibly also to monitor disease progression or regression after drug therapy. Some biomarkers present in blood and urine are associated with injury at specific sites in the nephron, which could help to identify and localize the injury earlier and even understand the mechanism of the pathogenesis of disease progression.

Unlike conventional experimental rodent models of diabetic nephropathy, of which, the relevance to human pathophysiology and metabolic kinetics remains unclear, it has been well-recognized that

*Corresponding author: Yi-Xin (Jim) Wang, Senior Vice President, Crown Bioscience, Inc, Cardiovascular and Metabolic Diseases Research, 3375 Scott Blvd STE108, 67 Bacon Court, Santa Clara, California 95054, USA, Tel: +0019253246861 E-mail: yxwang2000@gmail.com

Received December 18, 2013; Accepted February 07, 2014; Published February 12, 2014

Citation: Guo S, Qian W, Du F, Wang B, Wang X, et al. (2014) Proteinuria in Cynomolgus macaques (Macaca fascicularis) with Spontaneously Developed Metabolic Disorder and Diabetes: Transcriptome Analysis of Biopsy Kidney. J Diabetes Metab 5: 334. doi:10.4172/2155-6156.1000334

Copyright: (c) 2014 Guo S, et al. This is an open-access article distributed under the terms of the Creative Commons Attribution License, which permits unrestricted use, distribution, and reproduction in any medium, provided the original author and source are credited. 
Citation: Guo S, Qian W, Du F, Wang B, Wang X, et al. (2014) Proteinuria in Cynomolgus macaques (Macaca fascicularis) with Spontaneously Developed Metabolic Disorder and Diabetes: Transcriptome Analysis of Biopsy Kidney. J Diabetes Metab 5: 334. doi:10.4172/21556156.1000334

the Non-Human Primate (NHP) model of diabetes and metabolic disorders is the most clinically translatable animal model that resembles all the disease characteristics in patients at different stages of metabolic disorder and diabetes, including the progressive development of the known complications, such as diabetic nephropathy, thus providing an ideal animal model for investigating the mechanisms underlying human T2DM and diabetic nephropathy as well as for pharmaceutical discovery, development and evaluation of novel therapeutic agents [8]. Although there are some reports including the present laboratory with detailed characterization of the NHP model of diabetes and metabolic disorders, the diabetic complications, especially diabetic nephropathy in NHPs and its underline molecular mechanisms have not yet been well characterized [8-11].

Therefore, the aims of the present study were to

1. Characterize 50 cynomolgus monkeys at different stages of spontaneously developed metabolic disorders and diabetes for nephropathy phenotypes, such as urinary albumin, protein, glucose and creatinine excretion, kidney functions, etc.;

2. Examine the histopathological changes in the diabetic nephropathy kidneys;

3. Select 12 representative monkeys under 3 categories, normal control $(\mathrm{N})$, pre-diabetic without albuminuria (Pre-DM) and diabetic with albuminuria (DM) for transcriptome analysis of kidney biopsy specimen.

The present study was the first detailed characterization of the diabetic nephropathy phenotypes and the kidney histopathological changes in NHPs and provided molecular insights into novel mechanisms of disease progression, potential new drug targets as well as specific diagnostic biomarkers.

\section{Research Design and Methods}

\section{Animals}

Fifty Cynomolgus macaques (Macaca fascicularis) of both genders were individually housed at Crown Bioscience Inc., Taicang, China in species appropriate cages in temperature-controlled rooms maintained at $23 \pm 3^{\circ} \mathrm{C}$ on a 12-hour light-dark cycle. They were fed normal primate chow containing $19 \%$ protein, $5 \%$ fat and $3.6 \%$ fiber (Shanghai Shilin Biotechnology Inc., Shanghai, China) twice daily and had free access to tap water. All animals were weight stable prior to study initiation and during the study. The monkeys classified as the DM were recently diagnosed early diabetic, weight stable, negative for urinary ketones, and have not yet received insulin and any other therapy. All of the experimental procedures were approved by the Institutional Animal Care and Use Committee (IACUC) and were performed in accordance with the Guide for the Care and Use of Laboratory Animals of the United States National Institutes of Health (NIH).

\section{Intravenous Glucose Tolerance Test (ivGTT)}

In the absence of well-defined criteria to classify diabetes and glucose intolerance in NHPs, we used fasting glucose concentration, hemoglobin A1c level and glucose clearance rate obtained from ivGTT to evaluate the metabolic stages of the monkeys according to an established protocol $[8,9]$. The experiment was carried out under light ketamine anesthesia initiated with $10 \mathrm{mg} / \mathrm{kg}$ intramuscularly and supplemented to maintain sedation during the entire experiment period. The glucose solution ( $50 \%$ dextrose, $0.25 \mathrm{~g} / \mathrm{kg}$ ) was administered intravenously in a single bolus over a $30 \mathrm{sec}$. Blood samples were obtained intravenously immediately before and at 3, 5, 7, 10, 15, 20 and $30 \mathrm{~min}$ after glucose administration. Glucose disappearance or clearance rate (Kglucose) was expressed as the rate of glucose being removed from the circulation with a one compartment kinetic model (glucose blood volume distribution/glucose concentration Area Under The Curve, AUC) calculated by the slope of a linear trend best matching the natural logarithm of the blood glucose concentrations at each time point, by fitting a straight line using the least square method with Microsoft Excel formula: LINEST (LN (blood glucose concentrations at each time point), 3, 5, 7, 10, 15, 20, $30 \mathrm{~min}$ ).

Based on the multiple basic metabolic characterizations, such as fasting glucose, hemoglobin Alc and glucose clearance rate (Kglucose) levels, the monkeys were divided into 3 distinct groups: metabolically normal $(\mathrm{N})$, prediabetic or dysmetabolic (Pre-DM), and overtly diabetic (DM).

\section{Renal functional measurements}

The 24 hour urine collection was carried out with the monkeys kept individually in a stainless steel metabolic cage with a separation net being placed between the cage and the urine collection tray (Suzhou Houhuang Animal laboratory Equipment Technology Co. Ltd, China) to prevent the feces and residual food from contaminating the urine. An intra-venous blood sample was taken on the next day after urine collection for the biochemistry measurements by a clinical analyzer (Roche, Cobas 601). Renal function was evaluated by the clinically relevant surrogate markers, Blood Urea Nitrogen (BUN) and serum creatinine (Screatinine) concentrations, as well as Estimated Glomerular Filtration Rate (eGFR) based on creatinine clearance with the equation of Ucreatinine/Screatinine $\times 1.73$ / body weight ${ }^{2 / 3} \times 0.118$, which is commonly used in the clinic as a "first line" test of kidney function [12-14].

\section{Histopathology}

The kidneys from representative normal and diabetic nephropathy monkeys sacrificed for other IACUC approved studies with no kidney toxicity were dissected through laparotomy, cut open along coronary axis, fixed in $10 \%$ formalin (Sinopharmand), paraffin embedded (Leica EG1150G), cut by a microtome (Leica RM2245) at $5 \mu \mathrm{m}$ thick, H\&E and PAS stained, examined under a microscope (Nikon, TI-DH) and the image was taken with a cool CCD camera.

\section{Percutaneous kidney biopsy}

Twelve monkeys, $3 \mathrm{~N}$, 3Pre-DM without albuminuria and 6DM with albuminuria, were selected for kidney biopsy under ketamine anesthesia after an overnight fast. A $18 \mathrm{G}$ semi-automatic biopsy needle was used to penetrate the inferior pole of the kidney under sonographic localization and guidance. Each biopsy session would get 4 core samples to obtain a total of $\sim 10 \mathrm{mg}$ renal specimen. At the end of the procedure, buprenorphine $(5 \sim 10 \mu \mathrm{g} / \mathrm{kg})$ was given subcutaneously for pain relief. The collected renal biopsy specimen was put into RNA Stabilization Reagent, RNA later (Qiagen, USA) immediately, from which total messenger RNA was extracted and hybridized on AffymetrixGeneChip ${ }^{\circledR}$ Rhesus Macaque Genome Array. It has been reported that the genome divergence between rhesus and cynomolgus macaque is only $0.34-0.40 \%$ [15].

\section{Transcriptome analysis of renal biopsy specimen}

A total of 14764 genes were profiled on the AffymetrixGeneChip ${ }^{\circledR}$ 
Citation: Guo S, Qian W, Du F, Wang B, Wang X, et al. (2014) Proteinuria in Cynomolgus macaques (Macaca fascicularis) with Spontaneously Developed Metabolic Disorder and Diabetes: Transcriptome Analysis of Biopsy Kidney. J Diabetes Metab 5: 334. doi:10.4172/21556156.1000334

Rhesus Macaque Genome Arrays, of which 11074 genes could be assigned unique gene symbols and were selected for further analysis. Gene centric expression values were obtained using the custom Cumulative Distribution Function (CDF) file Rhesus_MAmu_ ENTREZG (version 16.0.0) from Brain array [16]. The background correction was performed using Robust Multichip Average (RMA) algorithm and quantile normalization. The expression values of mRNA were $\log _{2}$-transformed. The multiple pair wise comparisons were carried out to detect the Differentially Expressed Genes (DEGs) using Significance Analysis of Microarrays (SAM) software. The threshold was set to 4 fold change of mRNA expression between the 2 categories. As illustrated in Figure 1, the below 5 pairs were compared to delineate respective effects of dysmetabolism, diabetes and/or nephropathy contributing to the changes of gene expression in the renal biopsy specimen.

1. $\mathrm{N}$ vs. DM for the contribution of diabetic nephropathy;

2. N vs. Pre-DM for the contribution of dysmetabolism without nephropathy;

3. N vs. Pre-DM \& DM for the contribution of dysmetabolism with and without nephropathy;

4. Pre-DM vs. DM for the contribution of nephropathy in the presence of dysmetabolism;

5. DM vs. N \& Pre-DM for the contribution of nephropathy only.

Gene Set Enrichment Analysis (GSEA) by IPA software (Ingenuity) was used to draw biological interpretation of DEGs including their relationship to biological networks, diseases, and canonical pathways.

\section{Data and statistical analyses}

The diabetic nephropathy phenotype data are presented as mean and standard error of the mean. Group means among different groups were compared using ANOVA with Fisher LSD pairwise post hoc comparisons. Statistical significance was set at $\mathrm{p}<0.05$.

\section{Results}

\section{Basic classification of the NHP colonies}

The monkeys were classified as 3 categories, N, Pre-DM, and DM based mainly on general characterization for the fasting blood glucose, HbAlc, and ivGTT parameters. As shown in Table 1, the normal monkeys were obtained from a young colony with lighter body weight compared to the pre-DM and DM ones, which could only be obtained from an older population. Both the average fasting blood glucose concentrations and $\mathrm{HbAlc}$ were similar between the $\mathrm{N}$ and preDM, but significantly higher in DM monkeys. The calculated glucose clearance rate (Kglucose) via ivGTT was significant lower in the pre$\mathrm{DM}$ and the lowest in DM compared to $\mathrm{N}$.

\section{Characterization of diabetic nephropathy phenotypes in the NHPs}

Both the Dvolume (Figure 2A) and Uvolume (Figure 2B) were significantly lower in the pre-DM than both $\mathrm{N}$ and DM monkeys with the average Dvolume being $~ 14 \%$ higher than Uvolume measured simultaneously on the same day in all 3 groups. The Dvolume and Uvolume were highly correlated with each other for the individuals (Figure 2D). The Ualbumin was significantly higher in the DM than both $\mathrm{N}$ and pre-DM groups (Figure $2 \mathrm{C}$ ). Since the Uvolume and
Ualbumin could be variable in different days, 2 repeated experiments were carried out by collecting $24 \mathrm{hr}$ urine at $\sim 6$ weeks apart. The mean values of both the Uvolume (Figure 2B) and Ualbumin (Figure 2C) in each group were not significantly different, and the values from each individual monkey were highly correlated between the 2 separate measurements (Figure 2E and $2 \mathrm{~F}$ ). These data demonstrated that the present method for measurements of total daily drinking and urine volumes as well as urinary albumin excretion were highly reproducible.

The Uglucose (Figure 3A) and Uprotein (Figure 3B), but not Ucreatinine (Figure $3 \mathrm{C}$ ), were significantly elevated only in the DM monkeys. However, the urinary albuminuria index ACR was

\begin{tabular}{|l|l|l|l|}
\hline Serial No. & Comparison & Effect & \#DEG \\
\hline 1 & N vs. DM & Diabetic nephropathy & $\mathbf{7 5}$ \\
\hline 2 & N vs. Pre-DM & Dysmetabolic without nephropathy & $\mathbf{6 6}$ \\
\hline 3 & N vs. Pre-DM \& DM & Dysmetabolic with/without nephropathy & $\mathbf{6 8}$ \\
\hline 4 & Pre-DM vs. DM & Nephropathy in the presence of dysmetabolism & $\mathbf{1}$ \\
\hline 5 & DM vs. N \& Pre-DM & Nephropathy only & $\mathbf{4}$ \\
\hline
\end{tabular}
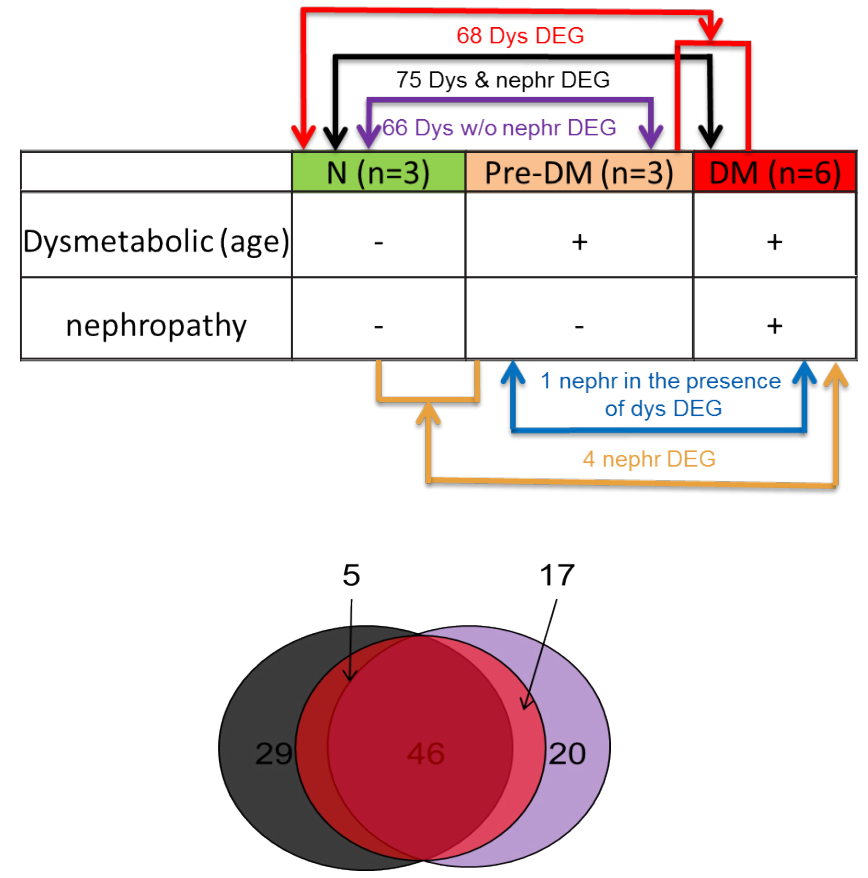

Figure 1: Comparative study design (top); the number of Differentially Expressed Genes (DEGs) detected for each corresponding comparison (middle) and overlap map for the DEGs (bottom).

\begin{tabular}{|l|c|c|c|}
\hline & Normal (N) & Pre-diabetic (Pre-DM) & Diabetic (DM) \\
\hline Subjects(n) & 11 & 19 & 20 \\
\hline Age (years) & $5.7 \pm 0.2$ & $15.1 \pm 0.6^{*}$ & $17.3 \pm 0.7^{\star} \#$ \\
\hline Body Weight (kg) & $5.5 \pm 0.5$ & $8.6 \pm 0.6^{*}$ & $7.5 \pm 0.7$ \\
\hline $\begin{array}{l}\text { Fasting Blood } \\
\text { Glucose (mg/dL) }\end{array}$ & $87 \pm 4.7$ & $91 \pm 2.8$ & $185 \pm 13.7^{*} \#$ \\
\hline HbA1c (\%) & $4.6 \pm 0.1$ & $5.1 \pm 0.4$ & $9.3 \pm 0.9^{*} \#$ \\
\hline Kglucose(g/L/min) & $2.9 \pm 0.26$ & $1.8 \pm 0.13^{*}$ & $1.2 \pm 0.15^{*} \#$ \\
\hline
\end{tabular}

*or \#: significantlydifferent from $\mathrm{N}$ or pre-DM group, respectively, $\mathrm{p}<0.05$, by ANOVA with Fisher (LSD) post hoc comparisons

Table 1: Descriptive characteristics (mean \pm standard error) 
Citation: Guo S, Qian W, Du F, Wang B, Wang X, et al. (2014) Proteinuria in Cynomolgus macaques (Macaca fascicularis) with Spontaneously Developed Metabolic Disorder and Diabetes: Transcriptome Analysis of Biopsy Kidney. J Diabetes Metab 5: 334. doi:10.4172/21556156.1000334

progressively elevated in the pre-DM and DM compared to $\mathrm{N}$ monkeys (Figure 3D). There were no significant changes in the renal functional indexes: BUN (Figure 4A) and Screatinine (Figure 4B) between the N and pre-DM, however, both were slightly but significantly reduced in DM group. The calculated eGFR was not significantly different among the 3 groups (Figure $4 \mathrm{C}$ ).

The correlation analysis was used to examine the relationship between several parameters. The Ualbumin was highly correlated with both Uprotein (Figure 5A) and ACR (Figure 5B) in all groups. When the FBG was $<150 \mathrm{mg} / \mathrm{dL}$ in most of the $\mathrm{N}$ and pre-DM monkeys, there was a minimal Uglucose $(<110 \mathrm{mg}$, Figure $5 \mathrm{D})$ and ACR $(<5 \mathrm{mg} / \mathrm{mmol})$ (Figure 5E). However, when the FBG was $>150 \mathrm{mg} / \mathrm{dL}$ threshold in most of the DM monkeys, it was positively correlated with both the Uglucose (Figure 5D) and ACR (Figure 5E). The Uglucose (Figure 5F), but not eGFR (Figure 5C) was modestly correlated with ACR.

\section{Histopathology of the kidney}

Histology of the kidneys from the representative DM monkeys with albuminuria was analyzed in comparison with a normal control one (Figure 6). The size of glomeruli was enlarged in the DM (Figures $6 \mathrm{D}$ and $6 \mathrm{E}$ ) compared with $\mathrm{N}$ kidney (Figures $6 \mathrm{~A}$ and $6 \mathrm{~B}$ ). Hemorrhage was frequently observed in the glomerulus of the DM kidney (Figure $6 \mathrm{E})$. Typical vacuolar degeneration of the tubules was shown in the DM kidney (Figure 6F). PAS staining revealed basement membrane thickening of the glomerular epithelial cells, mesangial matrix expansion
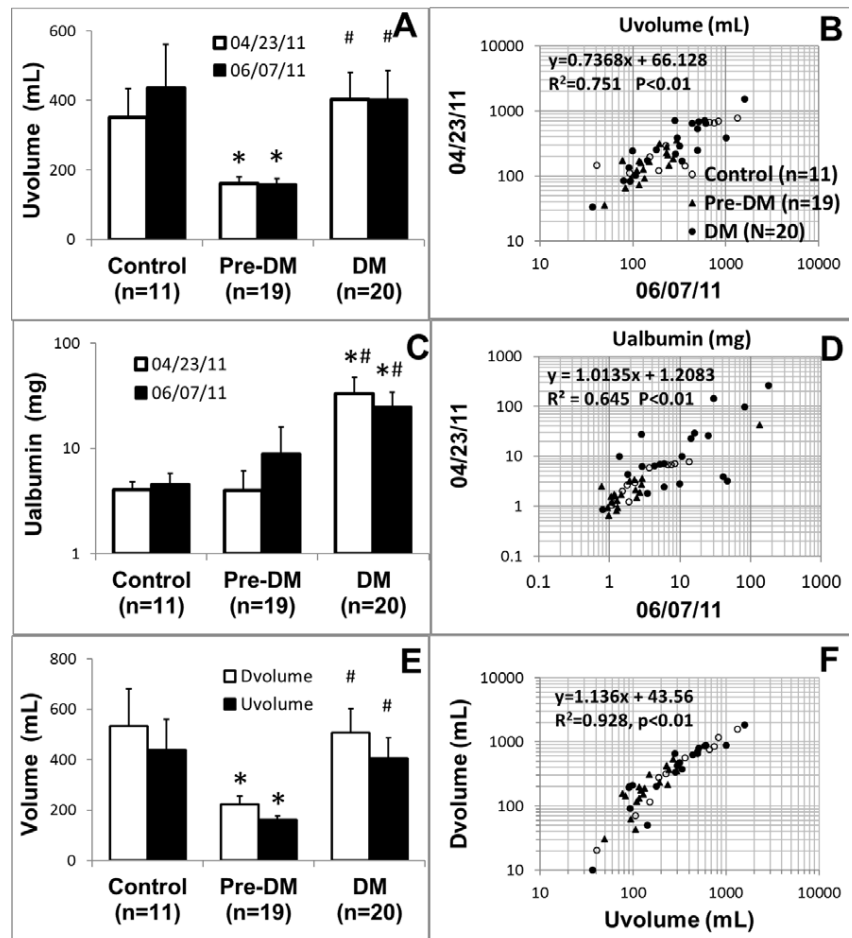

*or \#Significantly different from the normal control $(\mathrm{N})$ or pre-DM group, respectively, $\mathrm{p}<0.05$, by ANOVA with Fisher (LSD) post hoc comparisons

Figure 2: A. 24 hour total urine volume (Uvolume) measured repeatedly in 2 separate experiments; B. the correlation of the Uvolume between the 2 measurements; C. 24 hour total urine albumin excretion (Ualbumin) measured repeatedly in 2 experiments; D. the correlation of the Ualbumin between the 2 measurements; E. 24 hour total urine volume (Uvolume) measured simultaneously with the total drinking volume (Dvolume) in the same day; and $\mathrm{F}$. the correlation between the urine and drinking volume.

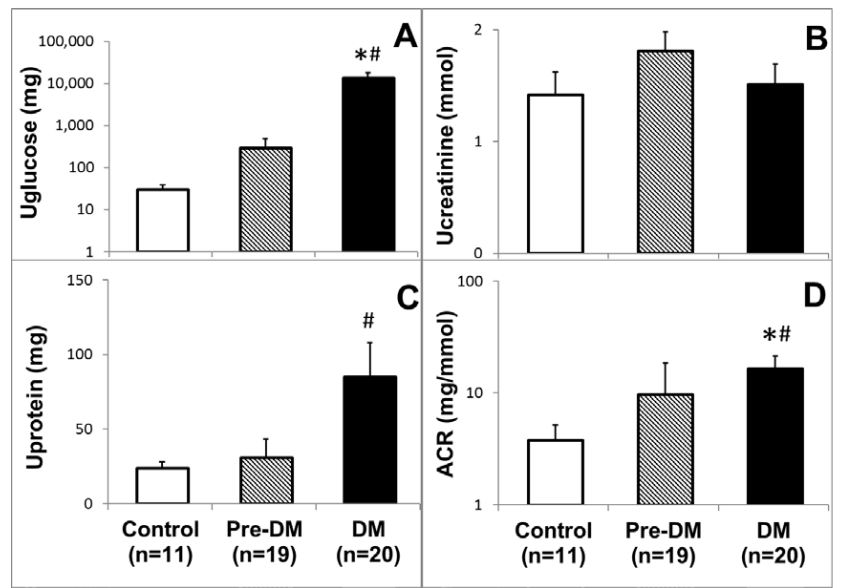

*or \# Significantly different from the normal control (N) or pre-DM group respectively, $\mathrm{p}<0.05$, by ANOVA with Fisher (LSD) post hoc comparisons

Figure 3: A.24 hour total urinary glucose (Uglucose); B. creatinine (Ucreatinine) C. protein (Uprotein) excretion; and D. urinary Albumin/Creatinine Ratio (ACR).
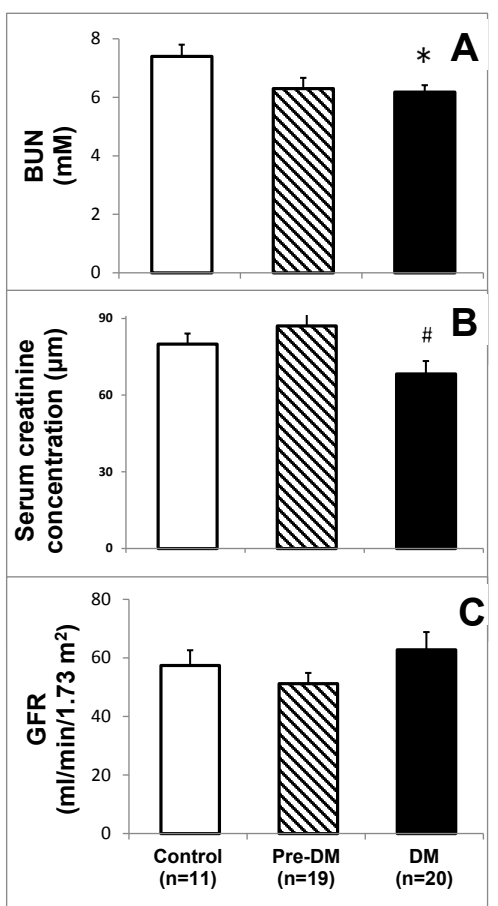

Figure 4: Renal functional surrogate markers measured by A. Blood Urea Nitrogen (BUN); B. serum creatinine concentrations; C. Glomerular Filtration Rate (GFR) calculated by creatinine clearance rate.

and glomerulosclerosis in the DM (Figure 6D) in comparison with $\mathrm{N}$ kidney (Figure 6A). These pathological characteristics are highly representative of the human diabetic nephropathy phenotype.

\section{Trascriptome analysis of the renal biopsy specimen}

Among the 11074 genes profiled and analyzed in the N, PreDM without albuminuria and DM with albuminuria NHPs selected for kidney biopsy, there are 95 unique DEGs detected with varying up- and down-regulation by a threshold of $>4$ folds between the 
Citation: Guo S, Qian W, Du F, Wang B, Wang X, et al. (2014) Proteinuria in Cynomolgus macaques (Macaca fascicularis) with Spontaneously Developed Metabolic Disorder and Diabetes: Transcriptome Analysis of Biopsy Kidney. J Diabetes Metab 5: 334. doi:10.4172/21556156.1000334

Page 5 of 8
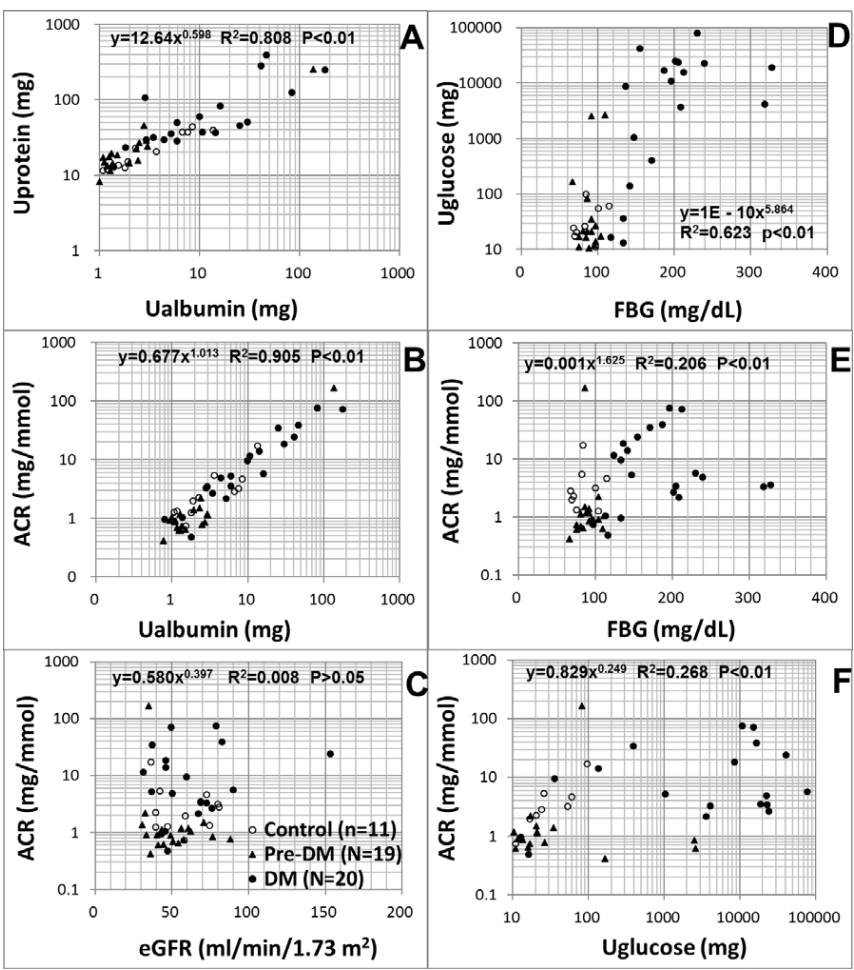

Figure 5: Correlation between A.ACR and blood glucose concentration; B. ACR and urinary albumin excretion; C. Urinary glucose excretion and blood glucose concentration; D. urinary protein and albumin excretion; E. ACR and estimated glomerular filtration rate; F. ACR and urinary glucose excretion.

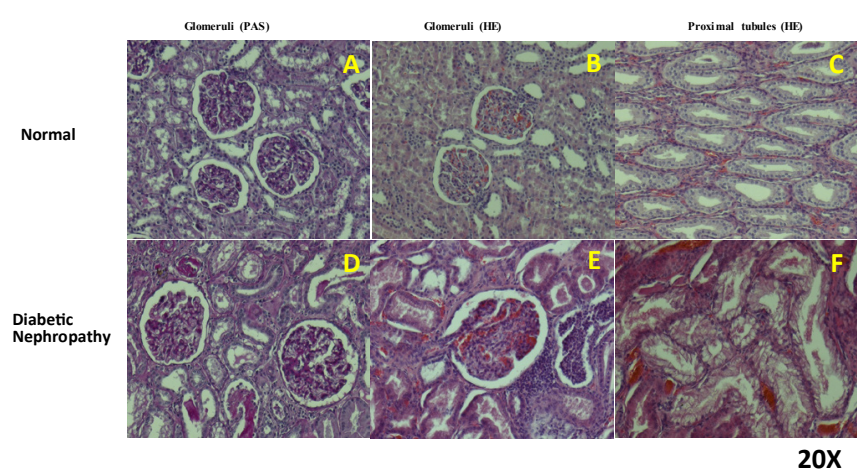

Figure 6: Histopathology of the kidneys from representative normal (N, top) or diabetic nephropathy (DM, bottom) monkeys with albuminuria for PAS (A \& D) and $H \& E(B, C, E \& F)$ staining.

comparison pair (Table S1), of which, at least 16 DEGs are related to kidney diseases and/or diabetes with their mRNA expression patterns presented in Figure 7. As shown in Figure 1, among the 95 DEGs, multiple pairwise comparisons by SAM software detected 75 diabetic nephropathy related DEGs (N and DM pair); 66 dysmetabolic without nephropathy DEGs (N and Pre-DM pair); 68 dsymetabolic specific DEGs ( $\mathrm{N}$ and dysmetabolic (Pre-DM\& DM) pair); but only LCT lactase as nephropathy specific gene (DM with albuminuria and PreDM without albuminuria pair); only 4 nephropathy specific DEGs (DM with albuminuria and all the other monkeys without albuminuria (N \& Pre-DM) pair), in which, HAVCR1, MMP7, and SPP1 were upregulated, and LCT down-regulated in the DM kidneys. Between the 75 diabetic nephropathy DEGs (N\&DM pair) and 66 dysmetabolic without albuminuria DEGs (N\& Pre-DM pair), there are 46 DEGs overlapping, thus, the 29 of 75 DEGs are unique only to N\& DM comparison, which may be more likely related to advanced disease stages including diabetes and nephropathy, while 3 of the 4 nephropathy DEGs (LCT, MMP7, and SPP1) found previously are members of the 29 DEGs, and HAVCR1 is 1 of the 46 overlapping common DEGs.

Using GSEA to analyze signaling pathways for the above 4 DEG encoded proteins, together with other 31 related proteins and protein complexes; it constructs a kidney failure, as well as renal, urological and inflammatory diseases related network (Figure 8). The most pivotal gene in this network is Tumor Necrosis Factor (TNF), which is a group of apoptosis-inducing cytokines, indicating that nephropathy is a disease closely related to inflammation and cell death.

\section{Discussion}

\section{Characterization of diabetic nephropathy phenotypes in NHPs}

Similar to early stages of diabetic nephropathy patients, the present study observed for the first time in cynomolgus monkeys that there is a progressive albuminuria and elevated ACR in those with severe diabetes, accompanied by aglycosuria, polydipsia and polyuria, with no significant changes in the Ucreatinine and Screatinin, BUN and eGFR, indicating that these monkeys may have impaired or leaky tubules but the kidney function is still largely preserved.

The great correlation between the Ualbumin and Uprotein in the present study indicates that these 2 parameters are parallel, both of which are the consequence of the leaky tubules allowing filtration of large molecules. However, as in many clinical reports, the Ualbumin is an earlier and more sensitive marker for tubular injury, which proceeds the detection of other Uprotein, thus has been commonly used in

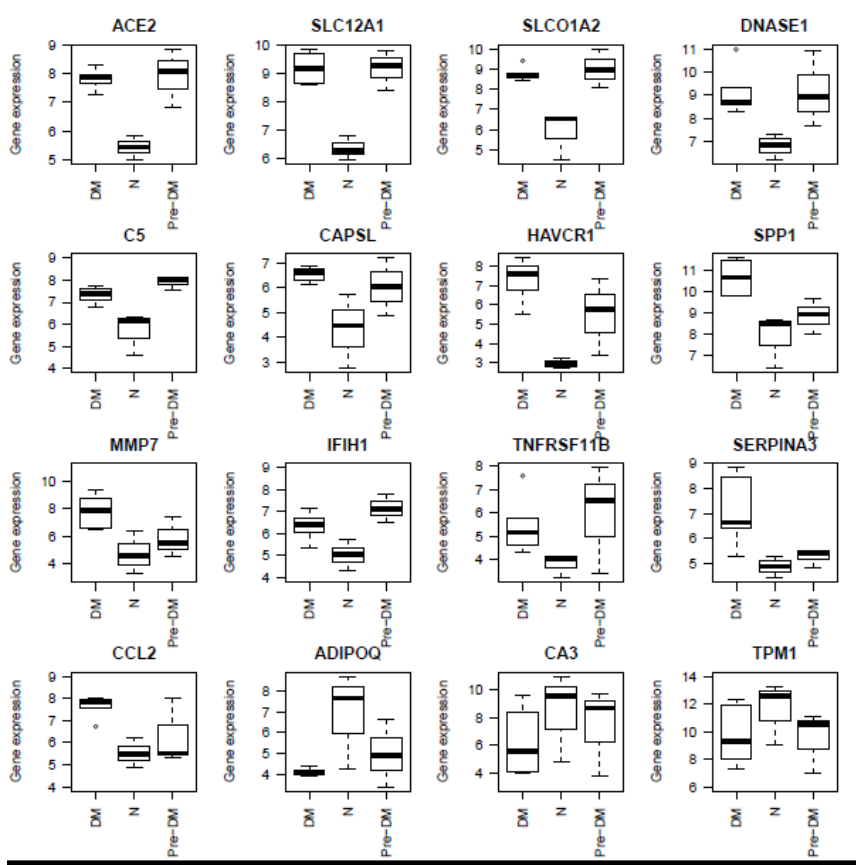

Figure 7: Kidney mRNA expression pattern of 16 DEGs known to be related to kidney diseases or diabetes in the kidneys from the N, Pre-DM without albuminuria and DM cynomolgus monkeys with albuminuria. 
Citation: Guo S, Qian W, Du F, Wang B, Wang X, et al. (2014) Proteinuria in Cynomolgus macaques (Macaca fascicularis) with Spontaneously Developed Metabolic Disorder and Diabetes: Transcriptome Analysis of Biopsy Kidney. J Diabetes Metab 5: 334. doi:10.4172/21556156.1000334

Page 6 of 8

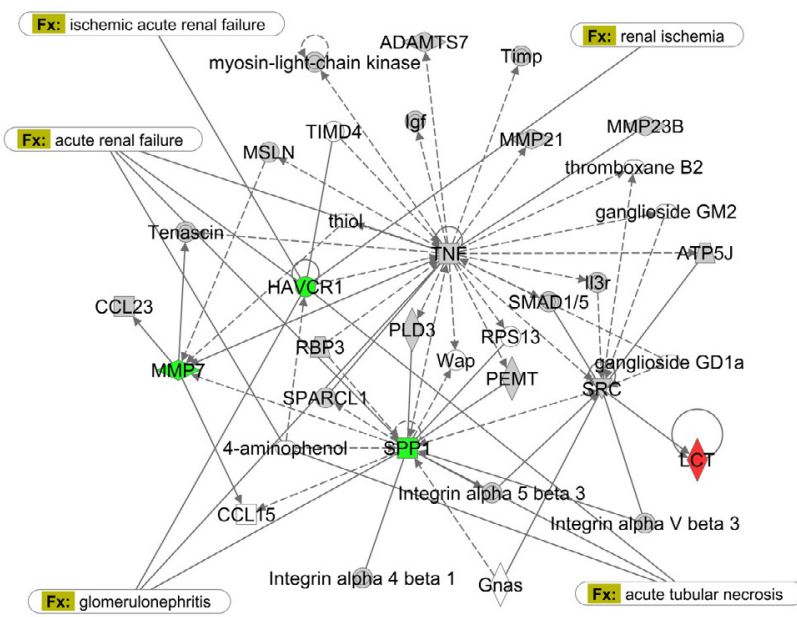

Figure 8: A biological network formed by 4 DEGs (colored) identified between the DM with albuminuria vs. N \& Pre-DM monkeys without albuminuria and other related genes and protein complexes (gray). Three genes (HAVCR1, MMP7, and SPP1) labeled in green have higher expression and 1 gene (LCT) labeled in red has lower expression pattern in the DM monkeys with albuminuria than those without (N \&Pre-DM).Solid lines refer to direct interactions and dashed lines indirect interactions between proteins. Genes participating in 5 renal diseases are noted as Fx in yellow. All relationships are experimentally validated reported in literatures.

clinic to diagnose and monitor early stage of diabetic nephropathy $[17,18]$. For example, in patients with Ualbumin even as low as 7 $\mathrm{mg}$, well below $30 \mathrm{mg}$ threshold for micro-albuminuria, it strongly correlates with increased risk of cardiovascular and cerebrovascular diseases, independent of the presence of other risk factors [18-20]. When Ualbumin is above $300 \mathrm{mg}$, it is generally indicative of diabetic nephropathy [21]. Currently, there is not a well-defined standard for the level of Ualbumin in monkeys with progress of metabolic disorders and diabetes. With the average body weight just $\sim 1 / 10$ in the cynomolgus monkeys $(5-15 \mathrm{~kg})$ compared to human $(\sim 75$ $\mathrm{kg}$ ), proportionally, it is reasonable to set Ualbumin at 8-20 mgfor micro-albuminuria and $>20 \mathrm{mg}$ for diabetic nephropathy. Although somewhat arbitrary, this criterion, however, fits to the characterization of the monkey colonies in the present and other studies [22]. Using this criteria, none of the normal monkeys had positive Ualbumin, only 1 pre-DM $>42 \mathrm{mg} ; 2 \mathrm{DM}$ with micro-albuminuria and $7 \mathrm{DM}$ with diabetic nephropathy, a distribution highly concentrated in the DM group (45\%). This observation is consistent with the report by Najafian et al. in spontaneously developed obese, dysmetabolic and diabetic rhesus monkeys [22].

The progressive nature of albuminuria is usually considered the result of escalating capillary endothelial dysfunction in the glomerular tuft while the basement membrane and underlying epithelial cells also play important roles [23]. The earliest detectable histopathogical change in the course of diabetic nephropathy is a glomerular hypertrophy (thickening in the glomerular basement membrane and capillary walls), and mild mesangial matrix expansion as shown in the present study in diabetic nephropathy cynomolgus as well as reported by Cusumano et al. in diabetic rhesus monkeys [24]. At this stage, the leaky tubules may enable the large amounts of serum albumin traverse the glomerular endothelium and associated structures, thus filtrate into the urine. But the 2 separate functions, impaired tubular uptake and compromised degradation may also independently prevent filtered albumin from reabsorption, thus, causing micro- and macro-albuminuria [25]. The compromised degradation may be the dysfunctional pathway for microalbuminuria before the global GFR is significantly reduced [17]. Instead, hyperglycemia may even result in kidney hemodynamic changes, leading to glomerular hyper-filtration in early stage of diabetes, which is consistent with the current observation that a slight, but not significant increase in eGFR, which in turn leading to a slight, but significant lower in both BUN and Screatinine in the DM monkeys (Figure 4) [26]. As diabetic nephropathy progresses, increasing numbers of glomeruli are damaged with progressive nodular glomerulosclerosis (formation of nodules in the glomerular tuft and angiopathy of capillaries in the glomeruli), increase in mesangial cellularity and matrix, etc., which generally showed in a clinical kidney biopsy as well as demonstrated in both rhesus and cynomolgus monkeys with diabetic nephropathy in the present study [24].

The present data further demonstrated that in the DM monkeys, the level of hyperglycemia is correlated with the severity of glucosuria (Figure 5D) and ACR (Figure 5E), indicating that hyperglycemia could contribute to the pathogenesis of nephropathy. There is no correlation between eGFR and ACR (Figure 5C) because these monkeys are still in the early stage of diabetic nephropathy with the renal function not significantly compromised yet. The increased Uvolume is caused by the osmotic effect of glycosuria in the DM compared to age-matched PreDM group. The relatively high Uvolume in the $\mathrm{N}$ monkeys is probably due to the much greater physical activities in the young population that is not compatible with the Pre-DM and DM population whose age is much older with much less physical activities, which may account for the less demand for drinking water, thus, less Uvolume in the Pre-DM group.

\section{Transcriptome analysis of the gene expressions of the kidney biopsy specimen}

The transcriptome analysis for the kidney biopsy tissues from 3 distinct groups in the present study detected 95 DEGs (Table $\mathrm{S} 1$ ), in which, at least 16 genes are found to be related to kidney diseases or diabetes (Figure 7). As showed in Table 2, specifically, HAVCR1 (KIM1) is involved in acute tubular necrosis and kidney injury, adriamicin-induced nephropathy, mesangial proliferative glomerulonephritis, polycystic kidney disease, anti-thymocyte serum nephritis, chromophobe renal cancer, protein overload nephropathy, renal ischemia, glomerulonephritis, proteinuria, etc. [27,28]. SPP1 is involved in acute tubular necrosis, acute kidney injury, polycystic kidney disease, systemic lupus erythematosus with renal impairment, proteinuria, etc. [28,29]. MMP7 is involved in hydronephrosis [30]. ACE2 is related to the thickness of basement membrane, which is a typical change in diabetic nephropathy patients, and SLCO1A2, CA3, SERPINA3 and TPM1 are related to nephropathy [31]. SLCO1A2 is associated with proximal tubular toxicity in male rat [32]. CA3 is associated with hypercalciuria, and an inhibitor of human CA3 protein (Chlorthalidone) has been approved for the treatment of hypercalciuria [33]. SERPINA3 in kidney is associated with acute renal allograft rejection in human [27]. ATP1A2, IFIH1, TNFRSF11B, ADIPOQ, SPP1, CA3, CCL2, SERPINA3, and CAPSL are involved in diabetes mellitus [34]. Clustering the mRNA expression values of these 16 DEGs separates diabetic nephropathy and normal monkeys perfectly, while Pre-DM monkeys spread in between (Figure 9), which is probably due to Pre-DM having different disease severity, spreading from the early to more advanced stages close to, develop nephropathy. Since the Pre$\mathrm{DM}$ and DM are in different stages in the progress of dysmetabolism 
Citation: Guo S, Qian W, Du F, Wang B, Wang X, et al. (2014) Proteinuria in Cynomolgus macaques (Macaca fascicularis) with Spontaneously Developed Metabolic Disorder and Diabetes: Transcriptome Analysis of Biopsy Kidney. J Diabetes Metab 5: 334. doi:10.4172/21556156.1000334

Page 7 of 8

\begin{tabular}{|c|c|c|}
\hline Genes & p-Value & Function Annotation \\
\hline \multicolumn{3}{|l|}{ Kidney Failure } \\
\hline HAVCR1,SPP1 & 4.22E-04 & acute tubular necrosis \\
\hline ADIPOQ,HAVCR1,SLC12A1,SPP1 & 1.06E-02 & renal failure \\
\hline DNASE1,SLC12A1 & 2.17E-02 & renal failure \\
\hline HAVCR1 & 4.46E-02 & ischemic acute renal failure \\
\hline ADIPOQ & 1.93E-01 & interstitial fibrosis of kidney \\
\hline \multicolumn{3}{|c|}{ Renal Hydronephrosis and Necrosis/Cell Death } \\
\hline CCL2,MMP7,SLC12A1,TNFRSF11B & 6.90E-04 & hydronephrosis \\
\hline DNASE1,SPP1 & 1.79E-03 & necrosis of renal tubule \\
\hline SPP1 & 1.00E-00 & apoptosis of kidney cell lines \\
\hline \multicolumn{3}{|l|}{ Renal Inflammation } \\
\hline C5,DNASE1,HAVCR1,IFIH1,SPP1 & 3.34E-03 & glomerulonephritis \\
\hline HAVCR1 & 4.55E-03 & $\begin{array}{l}\text { Mesangialproliferativeglo- } \\
\text { merulonephritis }\end{array}$ \\
\hline HAVCR1 & 9.08E-03 & anti-thymocyte serum nephritis \\
\hline SPP1 & 2.25E-02 & $\begin{array}{l}\text { experimental crescentic } \\
\text { glomerulonephritis }\end{array}$ \\
\hline HAVCR1 & 7.46E-02 & $\begin{array}{l}\text { membranous } \\
\text { glomerulonephritis }\end{array}$ \\
\hline HAVCR1 & 7.88E-02 & focal glomerulonephritis \\
\hline \multicolumn{3}{|l|}{ Renal Nephritis } \\
\hline C5,DNASE1,HAVCR1,IFIH1,SPP1 & 3.34E-03 & glomerulonephritis \\
\hline HAVCR1 & 4.55E-03 & $\begin{array}{l}\text { mesangial proliferative } \\
\text { glomerulonephritis }\end{array}$ \\
\hline HAVCR1 & 9.08E-03 & anti-thymocyte serum nephritis \\
\hline SPP1 & 2.25E-02 & $\begin{array}{l}\text { experimental crescentic } \\
\text { glomerulonephritis }\end{array}$ \\
\hline HAVCR1 & 7.46E-02 & $\begin{array}{l}\text { membranous } \\
\text { glomerulonephritis }\end{array}$ \\
\hline HAVCR1 & 7.88E-02 & focal glomerulonephritis \\
\hline
\end{tabular}

Table 2: Genes that are related to kidney disease.

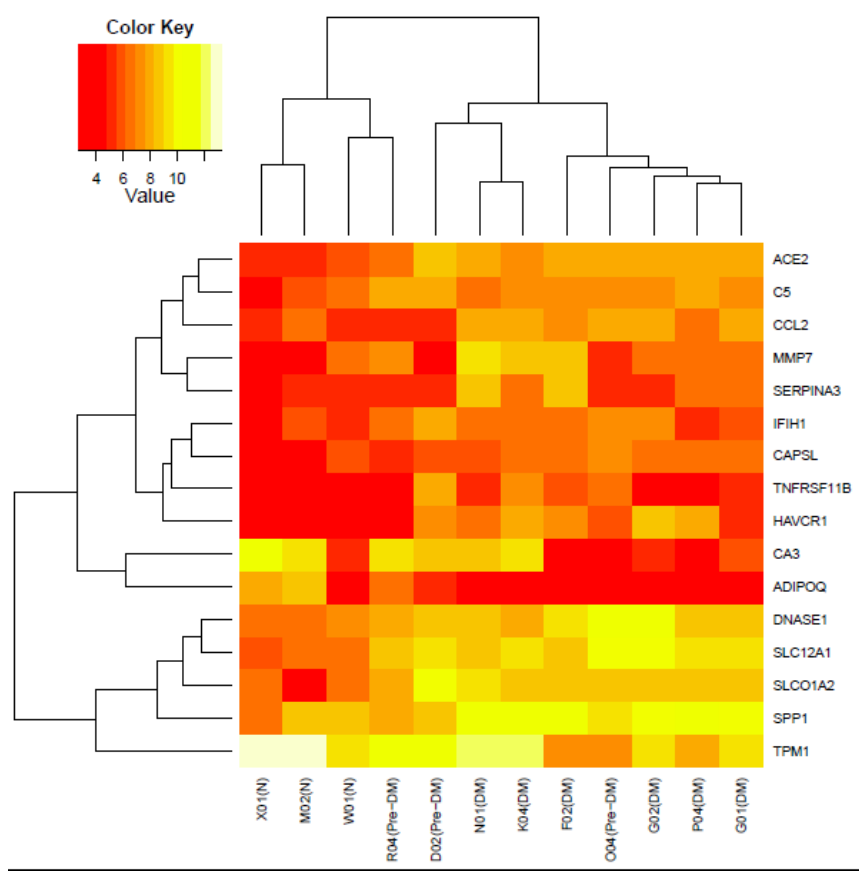

Figure 9: Clustering of 12 monkeys by the mRNA expression values of 16 DEGs known to be related to kidney diseases or diabetes.

and diabetes, the common genes related to the dysmetabolism are likely involved in the disease progression.
The 5 top canonical pathways significantly enriched in the 95 diabetic DEGs are the signaling pathways for calcium (ATP2A1, MYH4, MYL2, TNNC2, TNNT1 and TPM1), epithelial adherents junction (ACTN2, ACTN3, MYH4 and MYL), ILK (ACTN2, ACTN3, MYH4 and MYL2), VEGF (ACTN2, ACTN3, and EIF1AY) and Hepatic Fibrosis/Hepatic Stellate Cell Activation (CCL2, MYH4, MYL2, and TNFRSF11B). Among the biomarkers for progression and regression of diabetic nephropathy, only HAVCR1 (KIM-1) and SPP1 (Osteopontin) have shown significantly changed expression in diabetic nephropathy by our test criteria. A similar study of transcriptome analysis was reported in human diabetic kidney disease with the pathway analysis that in the glomeruli the regulation of $\mathrm{R}$ as homolog gene family member A, Cdc42, integrin, integrin-linked kinase, and VEGF signaling was highlighted, while in the tubule interstitial compartment, inflammation-related pathways was strong enriched [35]. The canonical complement signaling pathway was determined to be statistically differentially regulated in both glomeruli and tubuli and was associated with increased glomerulosclerosis in patients with diabetic kidney disease.

In summary, the present study demonstrated in the first time that spontaneously occurring dysmetabolic and diabetic cynomolgus monkeys developed similar diabetic nephropathy phenotypes as in human patients, such as hyperglycemia, polydipsia, polyuria, glucosuria, albuminuria, proteinuria, etc., however, with eGFR still remaining normal in the current colony. The histopathological examination also detected tubular injury without overlay glomerular destruction, which is consistent with that in patients with early stage of diabetic nephropathy. Transcriptome analysis of the kidney biopsy specimens revealed that the DEGs amongst $\mathrm{N}$, pre-DM and DM monkeys are highly related to kidney diseases and/or diabetes with signaling pathways constructing a kidney failure, renal, urological, and inflammatory diseases related network, in which the most pivotal gene is Tumor Necrosis Factor (TNF), indicating that nephropathy is a disease closely related to inflammation and cell death. Therefore, the present study demonstrated that NHP model of diabetic nephropathy had the same features as that in diabetic nephropathy patients with similar molecular, cellular, biochemical and pathophysiology mechanisms, thus, can be used as the most clinically translatable model for testing novel therapeutics for both the efficacy and potential adverse responses as well as identifying novel gene signatures for mechanism based diagnostic and therapeutic targets.

\section{Acknowledgements}

1. YXW took charge of the study design, results interpretation and manuscript writing, has full access to all the data in the study and is responsible for the integrity and accuracy of the experimental procedures and data analysis.

2. MB designed and performed the gene profiling experiment, data analysis manuscript writing and discussion.

3. FLD, BDW, XLW and YPF performed the experiment. XLW also performed data analysis and manuscript writing.

4. SG and WBQ performed the transcriptome data analysis and manuscript writing.

5. $Y X C$ and $X C$ performed histopathology analysis.

6. FMG, MS, KC, YFX and YXC involved in manuscript writing and discussion.

7. All the authors are the employees of Crown Bioscience, Inc. No potential conflicts of interest relevant to this article were reported.

8. Part of the data in this manuscript have been presented in 2013 ADA meeting and the abstract has been subsequently published in the proceedings of Diabetes Journal.

9. The authors gratefully acknowledge the excellent technical assistance of Shao $\mathrm{QM}$, ZengL and other vivarium staffs for their professionally caring of the HNPs and assisting in the experiments. 
Citation: Guo S, Qian W, Du F, Wang B, Wang X, et al. (2014) Proteinuria in Cynomolgus macaques (Macaca fascicularis) with Spontaneously Developed Metabolic Disorder and Diabetes: Transcriptome Analysis of Biopsy Kidney. J Diabetes Metab 5: 334. doi:10.4172/21556156.1000334

\section{References}

1. Moriya T, Tanaka S, Kawasaki R, Ohashi Y, Akanuma Y, et al. (2013) Diabetic retinopathy and microalbuminuria can predict macroalbuminuria and renal function decline in Japanese type 2 diabetic patients: Japan Diabetes Complications Study. Diabetes Care 36: 2803-2809.

2. Ruggenenti P, Fassi A, llieva A, lliev IP, Chiurchiu C, et al. (2011) Effects of verapamil added-on trandolapril therapy in hypertensive type 2 diabetes patients with microalbuminuria: the BENEDICT-B randomized trial. J Hypertens 29: $207-216$.

3. De Cosmo S, Motterlini N, Prudente S, Pellegrini F, Trevisan R, et al. (2009) Impact of the PPAR-gamma2 Pro12Ala polymorphism and ACE inhibitor therapy on new-onset microalbuminuria in type 2 diabetes: evidence from BENEDICT. Diabetes 58: 2920-2929.

4. Ritz E, Viberti GC, Ruilope LM, Rabelink AJ, Izzo JL Jr, et al. (2010) Determinants of urinary albumin excretion within the normal range in patients with type 2 diabetes: the Randomised Olmesartan and Diabetes Microalbuminuria Prevention (ROADMAP) study. Diabetologia 53: 49-57.

5. Menne J, Izzo JL Jr, Ito S, Januszewicz A, Katayama S, et al. (2012) Prevention of microalbuminuria in patients with type 2 diabetes and hypertension. $J$ Hypertens 30: 811-818.

6. Haller H, Viberti GC, Mimran A, Remuzzi G, Rabelink AJ, et al. (2006) Preventing microalbuminuria in patients with diabetes: rationale and design of the Randomised Olmesartan and Diabetes Microalbuminuria Prevention (ROADMAP) study. J Hypertens 24: 403-408.

7. Haller H, Ito S, Izzo JL Jr, Januszewicz A, Katayama S, et al. (2011) Olmesartan for the delay or prevention of microalbuminuria in type 2 diabetes. $\mathrm{N}$ Engl $\mathrm{J} \mathrm{Med}$ 364: 907-917.

8. Hansen BC (2012) Investigation and treatment of type 2 diabetes in nonhuman primates. Methods Mol Biol 933: 177-185.

9. Hansen BC, Bodkin NL (1993) Standardization of IVGTT. Importance of method used to calculate glucose disappearance. Diabetes Care 16: 847.

10. Wang X, Hansen BC, Shi D, Fang Y, Du F, et al. (2013) Quantification of $\hat{i}^{2}$ cell insulin secretory function using a graded glucose infusion with C-peptide deconvolution in dysmetabolic, and diabetic cynomolgus monkeys. Diabeto Metab Syndr 5: 40

11. Xiao YF, Wang B, Wang X, Du F, Benzinou M, et al. (2013) Xylazine-induced reduction of tissue sensitivity to insulin leads to acute hyperglycemia in diabetic and normoglycemic monkeys. BMC Anesthesiol 13: 33.

12. Stevens LA, Schmid $\mathrm{CH}$, Zhang YL, Coresh J, Manzi J, et al. (2010) Development and validation of GFR-estimating equations using diabetes, transplant and weight. Nephrol Dial Transplant 25: 449-457.

13. Stevens LA, Manzi J, Levey AS, Chen J, Deysher AE, et al. (2007) Impact of creatinine calibration on performance of GFR estimating equations in a pooled individual patient database. Am J Kidney Dis 50: 21-35.

14. Stevens LA, Levey AS (2009) Measured GFR as a confirmatory test for estimated GFR. J Am Soc Nephrol 20: 2305-2313.

15. Yan G, Zhang G, Fang X, Zhang Y, Li C, et al. (2011) Genome sequencing and comparison of two nonhuman primate animal models, the cynomolgus and Chinese rhesus macaques. Nat Biotechnol 29: 1019-1023.

16. Dai M, Wang P, Boyd AD, Kostov G, Athey B, et al. (2005) Evolving gene/ transcript definitions significantly alter the interpretation of GeneChip data. Nucleic Acids Res 33: e175.

17. Russo LM, Sandoval RM, Campos SB, Molitoris BA, Comper WD, et al. (2009) Impaired tubular uptake explains albuminuria in early diabetic nephropathy. $\mathrm{J}$ Am Soc Nephrol 20: 489-494.
18. Zamora CR, Cubeddu LX (2009) Microalbuminuria: do we need a new threshold? J Hum Hypertens 23: 146-149.

19. Volpe $M$ (2008) Microalbuminuria screening in patients with hypertension recommendations for clinical practice. Int J Clin Pract 62: 97-108.

20. Ruggenenti P, Porrini E, Motterlini N, Perna A, Ilieva AP, et al. (2012) Measurable urinary albumin predicts cardiovascular risk among normoalbuminuric patients with type 2 diabetes. J Am Soc Nephrol 23: 1717-1724.

21. Molitch ME, DeFronzo RA, Franz MJ, Keane WF, Mogensen CE, et al. (2004) Nephropathy in diabetes. Diabetes Care 27: S79-83.

22. Najafian B, Masood A, Malloy PC, Campos A, Hansen BC, et al. (2011) Glomerulopathy in spontaneously obese rhesus monkeys with type 2 diabetes: a stereological study. Diabetes Metab Res Rev 27: 341-347.

23. Satchell SC, Tooke JE (2008) What is the mechanism of microalbuminuria in diabetes: a role for the glomerular endothelium? Diabetologia 51: 714-725

24. Cusumano AM, Bodkin NL, Hansen BC, lotti R, Owens J, et al. (2002) Glomerular hypertrophy is associated with hyperinsulinemia and precedes overt diabetes in aging rhesus monkeys. Am J Kidney Dis 40: 1075-1085.

25. Comper WD, Hilliard LM, Nikolic-Paterson DJ, Russo LM (2008) Diseasedependent mechanisms of albuminuria. Am J Physiol Renal Physiol 295: F1589-1600.

26. Sallstrom J, Eriksson T, Fredholm BB, Persson AE, Palm F (2013) Inhibition of sodium-linked glucose reabsorption normalizes diabetes-induced glomerular hyperfiltration in conscious adenosine A -receptor deficient mice. Acta Physiol (Oxf).

27. Goligorsky MS, Addabbo F, O'Riordan E (2007) Diagnostic potential of urine proteome: a broken mirror of renal diseases. J Am Soc Nephrol 18: 2233-2239.

28. Vaidya VS, Ferguson MA, Bonventre JV (2008) Biomarkers of acute kidney injury. Annu Rev Pharmacol Toxicol 48: 463-493.

29. Xie Y, Nishi S, Iguchi S, Imai N, Sakatsume M, et al. (2001) Expression of osteopontin in gentamicin-induced acute tubular necrosis and its recovery process. Kidney Int 59: 959-974.

30. Henger A, Kretzler M, Doran P, Bonrouhi M, Schmid H, et al. (2004) Gene expression fingerprints in human tubulointerstitial inflammation and fibrosis as prognostic markers of disease progression. Kidney Int 65: 904-917.

31. Wong DW, Oudit GY, Reich H, Kassiri Z, Zhou J, et al. (2007) Loss of angiotensin-converting enzyme-2 (Ace2) accelerates diabetic kidney injury. Am J Pathol 171: 438-451.

32. Thukral SK, Nordone PJ, Hu R, Sullivan L, Galambos E, et al. (2005) Prediction of nephrotoxicant action and identification of candidate toxicity-related biomarkers. Toxicol Pathol 33: 343-355.

33. Maren TH, Wiley CE (1968) The in vitro activity of sulfonamides against red cell carbonic anhydrases. Effect of ionic and substrate variation on the hydration reaction. J Med Chem 11: 228-232.

34. Wellcome Trust Case Control Consortium (2007) Genome-wide association study of 14,000 cases of seven common diseases and 3,000 shared controls. Nature 447: 661-678.

35. Woroniecka KI, Park AS, Mohtat D, Thomas DB, Pullman JM, et al. (2011) Transcriptome analysis of human diabetic kidney disease. Diabetes 60: 23542369
This article was originally published in a special issue, Diabetic Cardiovascular Complications handled by Editor(s). Dr. Zhengyuan Xia, University of Hong Kong, Hong Kong 